\title{
EDITORIAL
}

\section{EMERGING CASES OF MULTI DRUG RESISTANT TUBERCULOSIS}

\author{
Denise Utami Putri \\ Intership doctor at Bhayangkara Hospital Yogyakarta
}

Tuberculosis (TB) is one of communicable disease caused by Mycobacterium genus. It is widely spread throughout the world, with at least one third of people is estimated to possess latent TB, a state where infection occurs, but does not develop disease symptoms. ${ }^{1}$ Indonesia, world's $4^{\text {th }}$ most populous country, has the highest incidence of TB cases in South East Asia, and $2^{\text {nd }}$ highest globally. It affects more than 1 million people, and has caused more than 100,000 deaths during 2014.2

Since 2000, Indonesian government, along with the United Nations, has implemented development goals to be achieved in 2015, the Millennium Development Goals (MDGs). MDGs consist of eight targets to be achieved in 2015. Along with the development of the country, Indonesia has made remarkable progress on the MDGs. In some areas progress has been impressive, including in efforts to combat communicable diseases: malaria, HIV/AIDS, and also, TB. Indonesian government has paid attention to this wide spread infection by encouraging case detection and diagnosis, as well as disease prevention and curative method. However, some assignments still need to be done in this Post-MGDs era. In addition to the still high prevalence ratio among population, the number of new cases detected each year is still growing. There has also been emerging cases of drug-resistant $T B$, which raise concern that the disease becomes more difficult, if not impossible, to treat. In new TB cases diagnosed without complications, the treatment success rate was not able to reach 90\% in 2014. This was even lower in multi drug resistant (MDR) TB, which had only 54\% cure rate. ${ }^{2}$

Mycobacteria strains resistant to one or several Anti TB regimens can be elicited through several mechanisms, including late diagnosis, prolonged infection, and poor compliance to treatment. Mutations of Mycobacterium tuberculosis genes have also been detec. ${ }^{3}$ These mutations led to stronger bacteria capable of resisting drugs. Therefore, efforts in detecting potentially severe infection should be made. Case findings, diagnostic methods, early and prompt treatment are very important in managing and preventing MDR TB, and ever Extensively Drug Resistant (XDR) TB, which hopefully will lead to a better outcome of disease, and limit morbidity and mortality.

In this edition, we published a cross sectional study conducted in Provincial Lung Clinic in Yogyakarta, Indonesia, involving $33 \mathrm{~TB}$ patients prior to Anti TB treatment. Drug susceptibility tests was conducted, and PCR-based spoligotyping was used to analyze genotype of Mycobacteria.

\section{REFERENCES}

1. World Health Organization (WHO). Global tuberculosis report; Geneva 2013.

2. World Health Organization (WHO). Global tuberculosis report; Geneva 2015.

3. Davies, PDO. Drug-resistant Tuberculosis. Journal of the Royal Society of Medicine, vol 94:6; 2001. 